\title{
THREE-DIMENSIONAL MODELING OF INTENSE BUNCHED BEAMS IN RF ACCELERATORS AND SOURCES*
}

\author{
R. Bhatt, M. Hess, and C. Chen, Massachusetts Institute of Technology \\ Plasma Science and Fusion Center, Cambridge, MA 02139, USA
}

\begin{abstract}
A three-dimensional model of intense bunched beams is developed for cylindrical and corrugated conducting pipes. Updated comparisons between the current limits derived from this model and experimental data from PPM focusing klystrons are presented. An electrostatic Green's function is formulated for azimuthally symmetric slowwave structures. Analytical and numerical results are presented for the potential of a periodic bunched pencil beam in a small-rippled waveguide approximation.
\end{abstract}

\section{INTRODUCTION}

The modeling of intense charged-particle beams is an important subject in beam physics [1,2] and to the development of $\mathrm{rf}$ accelerators and the high-power microwave (HPM) sources [3] driving them.

Recent analytic and semi-analytic Green's function analyses to address these issues have resulted in confinement conditions for bunched pencil beams in round cylindrical pipes [4-7]. The model predicts beam losses, which have been measured in a number of periodic permanent magnet (PPM) focusing klystrons at SLAC and KEK [8-12].

In this paper, we present updated comparisons between theory [4-7] and the PPM klystron experiments at SLAC $[8,9,11]$ and KEK $[10,12]$, and describe our initial efforts to generalize the previous model [4-7] to slow-wave structures. In particular, we derive an electrostatic Green's function for an azimuthally symmetric cylindrical conductor with small-amplitude axial variations of its radius. Self-consistent electrostatic potentials are computed for a periodic bunched beam propagating offaxis in the pipe.

\section{UPDATED COMPARISONS BETWEEN THEORY AND PPM KLYSTRON DATA}

The theoretical results, based on a three-dimensional bunched beam model for a perfectly conducting circular pipe with a constant radius, predict a current limit for PPM focusing klystrons being developed for $\mathrm{TeV}$ linear collider applications. For $\alpha \equiv 2 \pi a f / \gamma_{b} \beta_{b} c<2$, the limiting current $I_{b}$ is approximately given by [4-7]

$$
\frac{8 c^{2} I_{b}}{\omega_{c, r m s}^{2} a^{2} I_{A}} \cong \frac{\alpha}{\pi},
$$

where $a$ is the beam tunnel radius, $f$ is the operating

*Research supported by DOE and AFOSR. frequency of the klystron, $\beta_{b} c$ is the dc beam velocity, $\gamma_{b}$ is the relativistic mass factor for the dc beam, $\omega_{c, r m s}$ is the rms cyclotron frequency associated with the PPM focusing field, and $I_{A}=17 \mathrm{kA} \times \gamma_{b} \beta_{b}$ is the Alfven current.

Figure 1 and Table 1 show updated comparisons between the predicted (solid line) and the experimental data points. From Table 1 and Fig. 1, it is evident that both the SLAC 50 MW X-Band XL-PPM klystron [8,9] and Toshiba/KEK 50 MW C-Band PPM klystron [10] operate below the current limit. This agrees with minimal beam losses observed in both experiments. On the other hand, the SLAC $75 \mathrm{MW}$ X-Band XP-1 [8,9] and XP-3 [11] PPM klystrons and the BINP/KEK $75 \mathrm{MW} X-B$ and PPM-1 klystron [12] all operate at or above the current limit, and observe significant beam losses.

\section{ELECTROSTATIC GREEN'S FUNCTION IN A SLOW-WAVE STRUCTURE}

As an initial effort to generalize the previous model [47] to slow-wave structures, we pose the problem of determining the electrostatic Green function in an azimuthally symmetric slow-wave structure.

Employing cylindrical coordinates $(r, \phi, z)$, the conducting surface of the waveguide is specified by $r=a(z)$, where the pipe radius, $a$, is a function of the axial coordinate. The Green's function is then determined by the solution of

$$
\left\{\begin{array}{l}
\nabla^{2} G\left(\mathbf{x} ; \mathbf{x}^{\prime}\right)=-4 \pi \delta\left(\mathbf{x}-\mathbf{x}^{\prime}\right) \\
G(r=a(z))=0
\end{array}\right.
$$

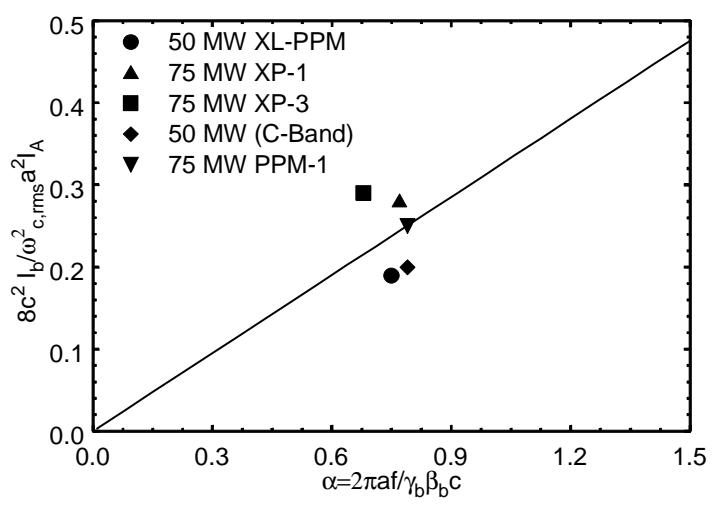

Figure 1: Comparison between theoretical current limit and experimental data for PPM klystrons. 
Table 1: Experimental parameters and theoretical current limits for PPM klystrons.

\begin{tabular}{|c|c|c|c|c|c|}
\hline PARAMETER & $\begin{array}{l}50 \text { MW XL- } \\
\text { PPM (SLAC) }\end{array}$ & $\begin{array}{c}75 \text { MW XP-1 } \\
\text { (SLAC) }\end{array}$ & $\begin{array}{c}75 \text { MW XP-3 } \\
\text { (SLAC) }\end{array}$ & $\begin{array}{c}50 \mathrm{MW} \\
\text { (Toshiba/KEK) }\end{array}$ & $\begin{array}{c}75 \text { MW PPM-1 } \\
\text { (BINP/KEK) }\end{array}$ \\
\hline$f(\mathrm{GHz})$ & 11.4 & 11.4 & 11.4 & 5.7 & 11.4 \\
\hline$I_{b}(\mathrm{~A})$ & 190 & 257 & 257 & 317 & 266 \\
\hline$\gamma_{b}$ & 1.83 & 1.96 & 1.96 & 1.69 & 1.94 \\
\hline$B_{r m s}(T)$ & 0.20 & 0.16 & 0.18 & 0.14 & 0.17 \\
\hline$a(\mathrm{~cm})$ & 0.48 & 0.54 & 0.48 & 0.90 & 0.55 \\
\hline$\alpha$ & 0.75 & 0.77 & 0.68 & 0.79 & 0.79 \\
\hline$\left.\frac{8 c^{2} I_{b}}{\omega_{c, \text { rms }}^{2} a^{2} I_{A}}\right|_{\exp }$ & 0.19 & 0.28 & 0.29 & 0.20 & 0.25 \\
\hline$\left.\frac{8 c^{2} I_{b}}{\omega_{c, r m s}^{2} a^{2} I_{A}}\right|_{c r i t}$ & 0.238 & 0.244 & 0.216 & 0.251 & 0.251 \\
\hline $\begin{array}{c}\text { Beam Power } \\
\text { Loss }\end{array}$ & $0.8 \%$ & $\begin{array}{r}\text { significant but } \\
\text { not measured }\end{array}$ & $\begin{array}{l}\text { significant but } \\
\text { not measured }\end{array}$ & $\begin{array}{c}\text { small but } \\
\text { not measured }\end{array}$ & $30 \%$ \\
\hline
\end{tabular}

This is reduced to a boundary value problem for Laplace's equation by separating the singular (free-space) and regular components of the Green's function and writing $G\left(\mathbf{x} ; \mathbf{x}^{\prime}\right)=\left|\mathbf{x}-\mathbf{x}^{\prime}\right|^{-1}+G_{R}\left(\mathbf{x} ; \mathbf{x}^{\prime}\right)$. This leaves

$$
\left\{\begin{array}{l}
\nabla^{2} G_{R}\left(\mathbf{x} ; \mathbf{x}^{\prime}\right)=0 \\
G(r=a(z))=f(\phi, z),
\end{array}\right.
$$

where the boundary function is defined by $f(\phi, z) \equiv-\left|\mathbf{x}(r=a)-\mathbf{x}^{\prime}\right|^{-1}$.

The regular part of the Green's function may be expanded in the cylindrical coordinate eigenfunctions of the Laplacian as

$$
G_{R}=\int_{-\infty}^{\infty} d \lambda \sum_{v=-\infty}^{\infty} e^{i \lambda z} e^{i v \phi} I_{v}(\lambda r) A_{\lambda v},
$$

where the amplitudes $A_{\lambda v}$ are determined by the boundary equation

$$
\begin{aligned}
G_{R}(a, \phi, z) & =f(\phi, z) \\
& =\int_{-\infty}^{\infty} d \lambda \sum_{v=-\infty}^{\infty} e^{i \lambda z} e^{i v \phi} I_{v}(\lambda a(z)) A_{\lambda v} .
\end{aligned}
$$

The standard procedure for determining the amplitudes $A_{\lambda_{v}}$ involves taking the Fourier transform of Eq. (5) and utilizing the orthogonality properties of the basis functions to isolate the amplitudes. This yields

$$
\tilde{f}_{\lambda^{\prime} v}=\frac{1}{2 \pi} \int_{-\infty}^{\infty} d z \int_{-\infty}^{\infty} d \lambda e^{i\left(\lambda-\lambda^{\prime}\right) z} A_{\lambda v} I_{v}(\lambda a(z)),
$$

where the Fourier transform of the boundary function is defined as

$$
\tilde{f}_{\lambda^{\prime} v^{\prime}}=\frac{1}{4 \pi^{2}} \int_{-\infty}^{\infty} d z \int_{-\pi}^{\pi} d \phi e^{-i \lambda^{\prime} z} e^{-i v^{\prime} \phi} f(\phi, z) .
$$

Note that for a constant $a(z)$ in Eq. (6), the $z$ integral reduces to a delta function, yielding the result for a straight cylinder [4]: $\tilde{f}_{\lambda v}=A_{\lambda v} I_{v}(\lambda a)$. For an arbitrary axial profile, Eq. (5) cannot be analytically inverted to obtain the amplitudes $A_{\lambda v}$.

\section{SMALL-RIPPLE APPROXIMATION}

The system does present an approximate analytic solution if the axially-varying conductor radius is approximately equal to its average value. We thus take the small ripple approximation, assuming that $a(z)=a_{0}+a_{1}(z)$, where $a_{1}(z)<<a_{0}$. Employing this approximation to Taylor expand the Bessel function appearing in Eq. (5) to first order in $a_{1} / a_{0}$ permits writing the transformed boundary function as $\tilde{f}_{\lambda^{\prime} v} \cong \tilde{f}_{\lambda^{\prime} v}^{0}+\tilde{f}_{\lambda^{\prime} v}^{1}$, where the result for the straight cylinder of constant radius $a_{0}$ is represented by $\tilde{f}_{\lambda^{\prime} v}^{0}=A_{\lambda^{\prime} v} I_{v}\left(\lambda^{\prime} a_{0}\right)$, and the first order correction due to the axially-varying wall radius is represented by

$$
\begin{aligned}
\tilde{f}_{\lambda^{\prime} v}^{1} & =\frac{1}{2 \pi} \int_{-\infty}^{\infty} d z \int_{-\infty}^{\infty} d \lambda e^{i\left(\lambda-\lambda^{\prime}\right) z} A_{\lambda v} \lambda a_{1}(z) I_{v}{ }^{\prime}\left(\lambda a_{0}\right) \\
& =\int_{-\infty}^{\infty} d \lambda A_{\lambda v} \lambda \tilde{a}_{1}\left(\lambda^{\prime}-\lambda\right) I_{v}^{\prime}\left(\lambda a_{0}\right),
\end{aligned}
$$

with the introduction of the Fourier transform of the wall ripple, defined by $\tilde{a}_{1}(\kappa)=\frac{1}{2 \pi} \int_{-\infty}^{\infty} a_{1}(z) e^{-i k z} d z$.

We shall henceforth in this paper assume a sinusoidal wall profile (see Fig. 2) given by $a_{1}(z)=\varepsilon a_{0} \cos (2 \pi z / \ell)$, with the understanding that an arbitrary (small-amplitude) wall profile may be constructed through an appropriate Fourier superposition of above modes. With this choice, the transformed boundary function becomes

$$
\begin{aligned}
\tilde{f}_{\lambda v}= & A_{\lambda v} I_{v}\left(\lambda a_{0}\right)+\frac{\varepsilon}{2} A_{\lambda-p, v} a_{0}(\lambda-p) I_{v}^{\prime}\left(a_{0}(\lambda-p)\right) \\
& +\frac{\varepsilon}{2} A_{\lambda+p, v} a_{0}(\lambda+p) I_{v}^{\prime}\left(a_{0}(\lambda+p)\right) .
\end{aligned}
$$

It is consistent with the small ripple approximation to use a first-order in $\varepsilon$ perturbation expansion to invert Eq. (9) for the amplitudes $A_{\lambda_{v}}$. Writing $A_{\lambda_{v}} \cong A_{\lambda_{v}}^{0}+\varepsilon A_{\lambda_{v}}^{1}$ and separately equating the coefficients of each order of $\varepsilon$ in Eq. (9) yields

$$
\begin{aligned}
A_{\lambda v} \cong & A_{\lambda v}^{0}-\frac{\varepsilon}{2} A_{\lambda-p, v}^{0} a_{0}(\lambda-p) \frac{I_{v}^{\prime}\left(a_{0}(\lambda-p)\right)}{I_{v}\left(a_{0} \lambda\right)} \\
& -\frac{\varepsilon}{2} A_{\lambda+p, v}^{0} a_{0}(\lambda+p) \frac{I_{v}^{\prime}\left(a_{0}(\lambda+p)\right)}{I_{v}\left(a_{0} \lambda\right)},
\end{aligned}
$$

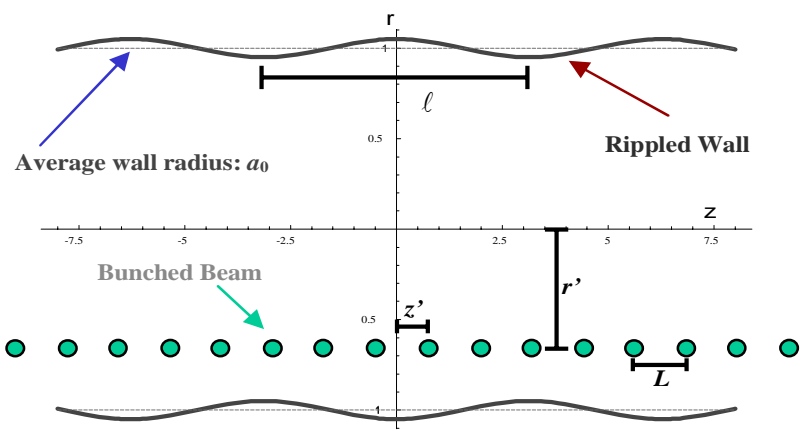

Figure 2: Bunched beam in a rippled-wall waveguide. 

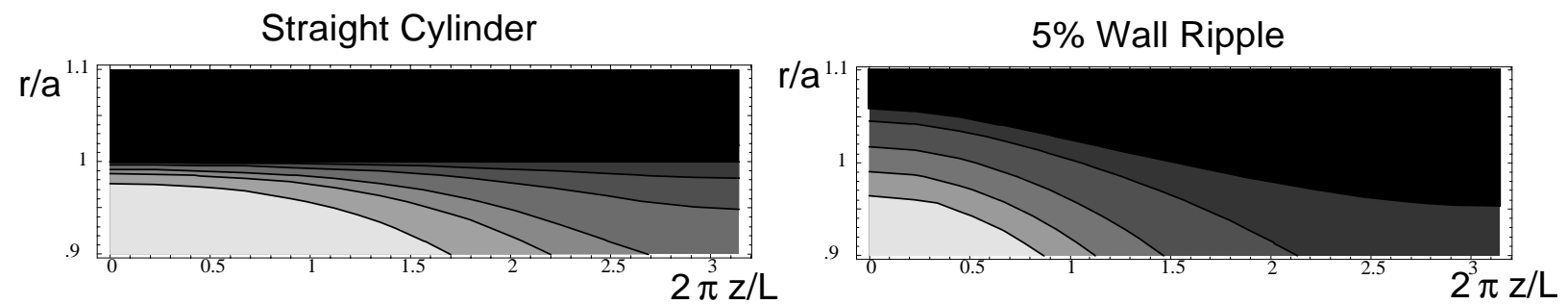

Figure 3: Equipotential curves for a bunched beam in a straight (left) and corrugated (right) cylinder.

where $A_{\lambda v}^{0}=\tilde{f}_{\lambda_{v}}\left[I_{v}\left(a_{0} \lambda\right)\right]^{-1}$.

All that remains is to determine the transformed boundary function, $\tilde{f}_{\lambda v}$. Employing a well-known Fourier-Bessel expansion [13] for the $1 / R$ potential, the boundary function may be written as

$$
\begin{aligned}
f(\phi, z) & =-\left|\mathbf{x}(r=a(z))-\mathbf{x}^{\prime}\right|^{-1} \\
& =-\frac{1}{\pi} \sum_{n=-\infty}^{\infty} \int_{-\infty}^{\infty} e^{i n\left(\phi-\phi^{\prime}\right)} e^{i k\left(z-z^{\prime}\right)} I_{n}\left(k r^{\prime}\right) K_{n}(k a(z)) d k,
\end{aligned}
$$

where we've assumed $r^{\prime}<a(z)$. Taking the Fourier transform as per Eq. (7) and making use of the small ripple approximation to expand the Bessel function yields

$$
\tilde{f}_{\lambda v}=-\frac{1}{\pi} e^{-i \lambda z^{\prime}} e^{-i v p^{\prime}}\left[\begin{array}{l}
I_{v}\left(\lambda r^{\prime}\right) K_{v}\left(\lambda a_{0}\right) \\
+\frac{\varepsilon}{2} e^{i p z^{\prime}} a_{0}(\lambda-p) I_{v}\left((\lambda-p) r^{\prime}\right) K_{V^{\prime}}\left((\lambda-p) a_{0}\right) \\
+\frac{\varepsilon}{2} e^{-i p z^{\prime}} a_{0}(\lambda+p) I_{v}\left((\lambda+p) r^{\prime}\right) K_{v}\left((\lambda+p) a_{0}\right)
\end{array}\right] .
$$

Combined with Eqs. (10) and (4), this result determines the electrostatic Green's function for an azimuthally symmetric pipe with small-amplitude axial variations in its radius, accurate to first order in $\varepsilon$.

Given the Green's function, the electrostatic potential may be computed for an arbitrary charge distribution. For purposes of comparison, it is useful to consider the bunched beam charge distribution of [4],

$$
\rho(r, \phi, z)=\frac{1}{r} \delta\left(r-r^{\prime}\right) \delta\left(\phi-\phi^{\prime}\right) \sum_{n=-\infty}^{\infty} \delta\left(z-z^{\prime}-n L\right) .
$$

This distribution (depicted in Fig. 2) represents a periodic bunched beam with bunch spacing $L$, displaced from the axis by a distance $r^{\prime}$, and with one bunch offset from the $z=0$ plane by a distance $z^{\prime}$. Integrating the Green's function over this distribution yields $\Phi(\mathbf{x})=\Phi^{0}(\mathbf{x})+\varepsilon \Phi^{1}(\mathbf{x})$, where the first term is the straight cylinder potential of [4],

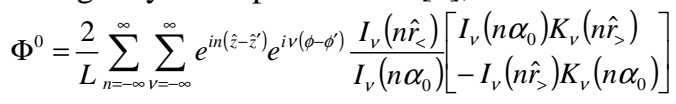

and the second term gives the correction due to a sinusoidal wall ripple,

$$
\Phi^{0}=\frac{1}{L} \sum_{n=-\infty}^{\infty} \sum_{V=-\infty}^{\infty} e^{i n\left(\hat{z}-\hat{z}^{\prime}\right)} e^{i v\left(\hat{\phi}-\hat{\phi}^{\prime}\right)}\left[\begin{array}{c}
e^{i \hat{k} \hat{p}} \frac{I_{v}\left(n \hat{r}^{\prime}\right) I_{v}((n-\hat{p}) \hat{r})}{I_{v}\left(n \alpha_{0}\right) I_{v}\left((n-\hat{p}) \alpha_{0}\right)} \\
+e^{-i \hat{p} \hat{p}} \frac{I_{v}\left(n \hat{r}^{\prime}\right) I_{v}((n+\hat{p}) \hat{r})}{I_{v}\left(n \alpha_{0}\right) I_{v}\left((n+\hat{p}) \alpha_{0}\right)}
\end{array}\right] .
$$

The quantities introduced above are defined as $\alpha_{0} \equiv 2 \pi a_{0} / L, \quad \hat{r} \equiv 2 \pi r / L, \quad \hat{r}^{\prime} \equiv 2 \pi r^{\prime} / L, \quad \hat{z} \equiv 2 \pi z / L$, $\hat{z}^{\prime} \equiv 2 \pi z^{\prime} / L, \quad \hat{p} \equiv L / \ell, \hat{r}_{<} \equiv \min \left(\hat{r}, \hat{r}^{\prime}\right), \hat{r}_{<} \equiv \max \left(\hat{r}, \hat{r}^{\prime}\right)$, and $a_{1}(z)=\varepsilon a_{0} \cos (2 \pi z / \ell)$.

Figure 3 shows equipotential curves in the plane of the charge for a bunched beam in both a straight and corrugated cylinder. Relevant parameters are $\alpha_{0}=2$, $\hat{r}^{\prime}=0.5, \quad \hat{z}^{\prime}=0, \hat{p}=1$, and $\varepsilon=0.05$. The black region (corresponding to the zero potential surface) conforms to the straight and sinusoidally rippled cylindrical walls, respectively.

\section{REFERENCES}

[1] M. Reiser, Theory and Design of Charged-Particle Beams (Wiley \& Sons, New York, 1994).

[2] R.C. Davidson, Physics of Nonneutral Plasmas (Addison-Wesley, Reading, MA, 1990).

[3] J. Benford and J. Swegle, High Power Microwaves (Artech, Boston, 1992).

[4] M. Hess and C. Chen, Phys. Plasmas 75206 (2000).

[5] M. Hess and C. Chen, Phys. Lett. A295, 305 (2002).

[6] M. Hess and C. Chen, Phys. Plasmas 9, 1422 (2002).

[7] M. Hess, MIT Ph.D. Thesis (2002).

[8] D. Sprehn, G. Caryotakis, E. Jongewaard, R.M. Phillips, in Proceedings of $19^{\text {th }}$ International Linac Conference (Argonne National Laboratory Report ANL-98/28), p.689.

[9] D. Sprehn, et al., in Intense Microwave Pulses VII, edited by H. E. Brandt, SPIE Proc. 4301 (2000), p. 132.

[10] Y. H. Chin, et al, in Proceedings of the Particle Accelerator Conference 2001, edited by P. W. Lucas and S. Webber, (2001), p. 3792.

[11] SLAC Website http://wwwproject.slac.stanford.edu/lc/local/MAC/OCT2001/C aryotakis\%20NLC\%20KLYSTRON\%20R.pdf

[12] H. Matsumoto, et al, in Proceedings of the Particle Accelerator Conference 2001, edited by P. W. Lucas and S. Webber, (2001), p. 993.

[13] Jackson, J., Classical Electrodynamics (Wiley, New York, 1962).

[14] Gradshteyn, I.S. and Ryzhik, I.M, Table of Integrals, Series, and Products, $5^{\text {th }}$ Edition (Academic Press, San Diego, 1994). 